\title{
Análisis estadístico de incendios forestales en la Comunitat Valenciana y modificación del índice de peligrosidad de incendios (PIF)
}

https://doi.org/10.31978/639-19-010-0.697

\author{
Cristina Franco Radín ${ }^{1}$ (radin@alumni.uv.es) \\ Adrián Revert Ferrero' ${ }^{1}$ (refea@alumni.uv.es) \\ Javier Mediavilla González² (jmediavillag@aemet.es) \\ José Ángel Núñez Mora² (jnunezm@aemet.es)
}

${ }^{1}$ Universidad de Valencia / Prácticas en AEMET

${ }^{2}$ AEMET / Delegación Territorial en la Comunidad Valenciana

\begin{abstract}
RESUMEN
En la Comunitat Valenciana se producen una gran cantidad de incendios forestales cada año, quemando algunos de ellos miles de hectáreas y ocasionando graves impactos. En el Grupo de Predicción y Vigilancia (GPV) de Valencia de la Agencia Estatal de Meteorología se elabora diariamente el indice de peligrosidad de incendios forestales, que es utilizado por el Centro de Emergencias de la Generalitat Valenciana para activar diariamente las alertas por incendios forestales.

Este estudio ha analizado estadísticamente la base de datos de incendios forestales de los años 2016 y 2017 en la Comunitat Valenciana en relación al índice PIF-GPV previsto para cada día. Una vez evaluado el funcionamiento del índice y con la finalidad de aumentar su precisión, se han introducido en su cálculo nuevas variables como la humedad del suelo y la precipitación registrada.
\end{abstract}

PALABRAS CLAVE: incendios forestales; índice PIG-GPV; predicción; prevención de incendios; balance hídrico.

\section{INTRODUCCIÓN}

El fuego es inherente a los ecosistemas mediterráneos: su vegetación es pirófita, es decir, se encuentra adaptada al fuego. Este se produce de forma natural por las descargas eléctricas. Pese a todo, durante las últimas décadas se ha constatado un aumento de la recurrencia de los incendios forestales y de su extensión debido a causas antrópicas. La mayoría se originan por negligencias y accidentes, pero también se producen de forma intencionada. En la Comunitat Valenciana se producen muchos incendios cada año, siendo la mayoría de poca extensión gracias a la rápida actuación de los medios. En ocasiones se producen grandes incendios que causan graves impactos, tanto para los ecosistemas como para la población y sus bienes.

El índice PIF-GPV se elabora diariamente en el Grupo de Predicción y Vigilancia (GPV) de Valencia de AEMET con la finalidad de asignar un nivel de peligrosidad de propagación de incendio basándose en las condiciones meteorológicas previstas. Este índice se predice para cada una de las siete zonas en las que se subdivide la Comunitat Valenciana según la zonificación establecida por el PREVIFOC (Plan sectorial de prevención contra incendios). A partir del índice PIF-GPV, Emergencias de la Generalitat Valenciana activa diariamente las alertas por incendios forestales, existiendo 3 niveles de alerta: alerta 1, peligro medio-bajo; alerta 2, peligro alto; y alerta 3, peligro extremo.

Una descripción del trabajo más pormenorizada puede consultarse en RADín et al. (2018). 


\section{OBJETIVOS Y METODOLOGÍA}

El objetivo del estudio ha sido el análisis estadístico de los incendios registrados y del índice pronosticado en el GPV con la finalidad de evaluarlo e introducir mejoras en su cálculo. Se ha utilizado la siguiente información, para los años 2016 y 2017 :

- Base de datos de incendios forestales de la Generalitat Valenciana.

- Base de datos del índice PIF-GPV elaborado por el GPV de Valencia.

Los parámetros estudiados han sido, para cada periodo considerado y en función del nivel de alerta:

- Número de incendios forestales.

- Superficie quemada (en hectáreas).

- Número de días de cada alerta.

- Número de incendios por día según el nivel de alerta: es un valor relativo, el cociente entre el número de incendios y el número de días con cada nivel de alerta. Los resultados globales se han calculado en base al número total de alertas activas en las 7 zonas PREVIFOC, de modo que el cociente se ha multiplicado por 7 para obtener un valor medio de todo el territorio.

- Superficie quemada por día según el nivel de alerta: es un valor relativo, el cociente entre la superficie total quemada y el número total de días con ese nivel de alerta. También se ha calculado el resultado global de la Comunitat Valenciana para obtener un valor medio.

- Superficie quemada por incendio: es el cociente entre la superficie quemada y el número de incendios.

Para la modificación del índice se han tenido en cuenta la humedad del suelo y las precipitaciones acumuladas semanales. El método ha consistido en la obtención de los productos del balance hídrico en forma de mapas diarios (desde el 01/01/2016 hasta el 31/12/2017) para extraer sus datos y, a posteriori, se ha realizado una correlación de dichos datos con los datos de incendios.

\section{RESULTADOS DEL ESTUDIO ESTADÍSTICO}

\subsection{Resultados globales del estudio estadístico}

Entre 2016 y 2017 se produjeron 690 incendios forestales en la Comunitat Valenciana, quemando un total de 8811,9 hectáreas. El número de incendios fue similar los dos años, pero en 2016 (año muy seco) se quemaron casi cuatro veces más hectáreas (6913,7 ha) que en 2017 (1898,3 ha).

a) Porcentaje de días con cada alerta

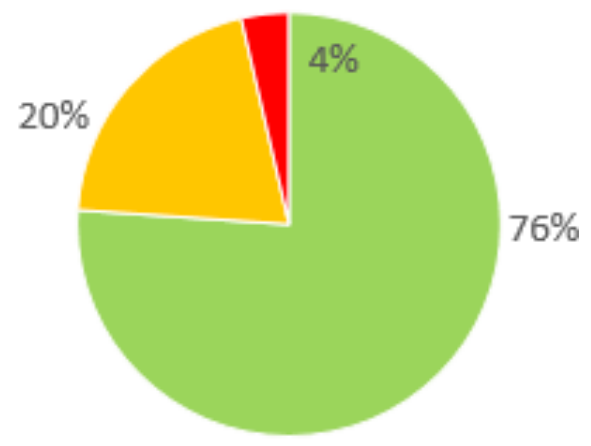

- Alerta 1 Alerta 2 Alerta 3 b)

\section{Porcentaje de superficie quemada}

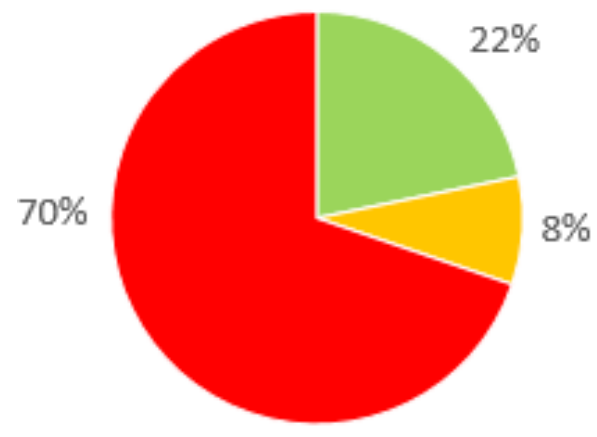

n Alerta 1 Alerta 2 alerta 3

Figura 1. a) Porcentaje de días de alerta. b) Porcentaje de superficie quemada según la alerta. 
Atendiendo a la figura 1, durante gran parte del año (76\%) predominó el nivel medio-bajo de peligrosidad, suponiendo el peligro alto un $20 \%$ y los días de peligro extremo tan solo un $4 \%$. En ese $4 \%$ de días se quemó el $70 \%$ de la superficie total incendiada en todo el territorio.
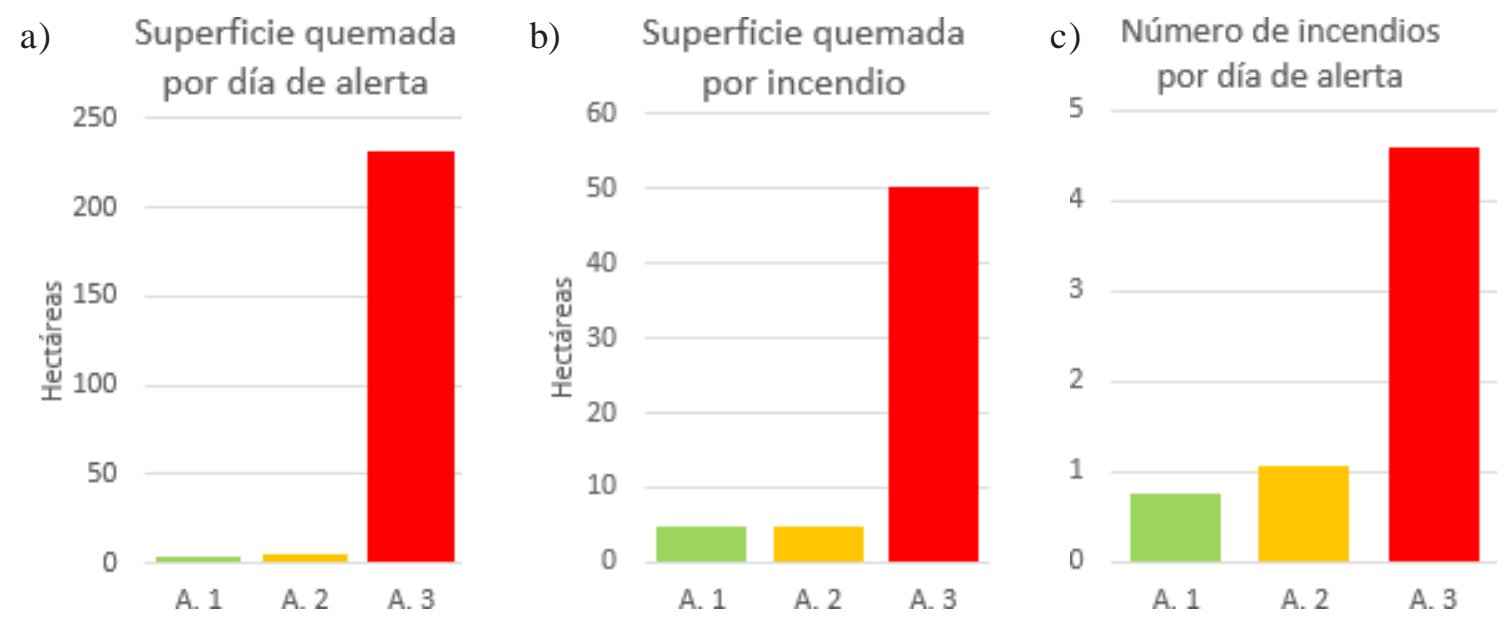

Figura 2. Estadísticas sobre la gravedad de los incendios. a) Superficie quemada por día de alerta.

b) Superficie quemada por incendio forestal. c) Número de incendios por día de alerta.

En la figura 2 se puede observar como los parámetros referidos a la gravedad de los incendios se incrementaron de forma muy notable en los días de alerta 3 . Mientras tanto, los valores de los días de alertas 1 y 2 fueron similares y muy inferiores a los registrados con peligro extremo.

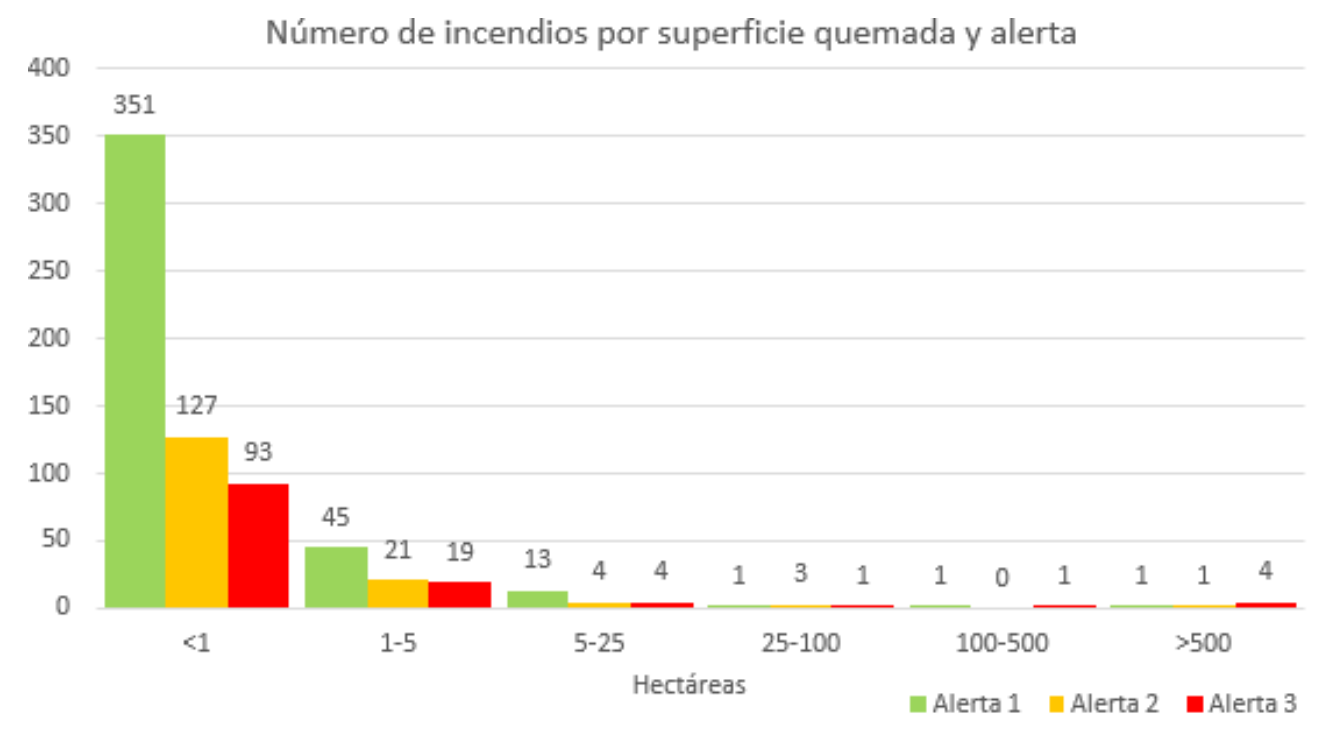

Figura 3. Número de incendios por superficie quemada y alerta.

Como se observa en la figura 3 , expresada en términos absolutos, la mayoría de los incendios que se produjeron fueron pequeños, el $80 \%$ quemaron menos de 1 ha. La mayor parte se registraron en días de alerta 1 (debido al alto porcentaje de días con dicha alerta), siendo muchas veces quemas agrícolas descontroladas en las que las condiciones meteorológicas poco favorables para su expansión y la rápida actuación de los medios de extinción evitaron incendios más extensos. En los grandes incendios (más de 500 ha) hubo un claro predominio de los fuegos producidos en días de alerta 3: fueron incendios de zonas forestales, generalmente montañosas, durante días de condiciones meteorológicas favorables para la propagación del fuego y, por tanto, adversas para su extinción, de manera que quemaron grandes superficies. 


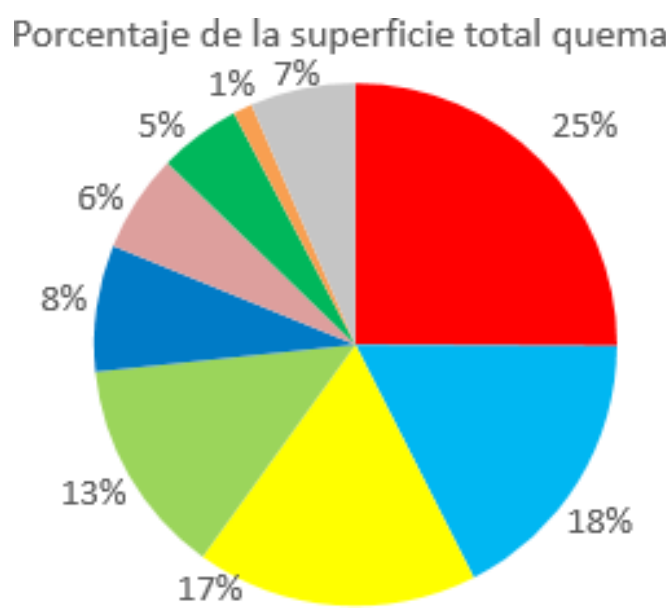

Figura 4.

Porcentaje de la superficie total quemada por los distintos incendios forestales.

\author{
- Carcaixent \\ - Chella \\ Artana \\ Gátova-Segorbe \\ - Benitatxell \\ Culla \\ - Bolulla \\ Xeresa \\ Resto de incendios
}

Muestra de la importancia de los grandes incendios en el total de hectáreas quemadas es que los 8 incendios más grandes de 2016 y 2017 quemaron una superficie trece veces mayor que los 682 incendios restantes, como bien refleja la figura 4.

Si se analizan los datos teniendo en cuenta la zonificación del PREVIFOC, se llega a la conclusión de que las zonas 3 y 5 son las que más incendios y de mayor extensión registraron. Esto se explica porque abarcan una gran superficie del interior, donde hay muchos espacios forestales. En cambio, las zonas 2, 4 y 6, que abarcan espacios litorales y prelitorales, registraron una superficie quemada muy pequeña, al ser zonas con menos superficie forestal. En todas las zonas se produjeron más incendios con alerta 1 (la predominante durante gran parte del año) pero se quemaron muchas más hectáreas con alerta 3, a excepción de las zonas 1N (incendio de Culla con alerta 2) y $1 \mathrm{~S}$ (incendio de Artana con alerta 1).

Por último, como se puede observar en el mapa de la figura 5, la mayor parte de los grandes incendios forestales se produjo en espacios montañosos prelitorales, durante los días de verano y estando activo el nivel 3 de alerta PIF-GPV. A lo largo de toda la Comunitat Valenciana se produjeron incendios de pequeña importancia, siendo menos propicias a los incendios en 2016 y 2017 las comarcas de Els Ports (CS), el Valle de Ayora-Cofrentes (V) y el Alto Vinalopó (A).

Figura 5.

Mapa de distribución de los incendios forestales en la Comunitat Valenciana en 2016 y 2017 según los niveles de alerta y la superficie quemada (círculos proporcionales).

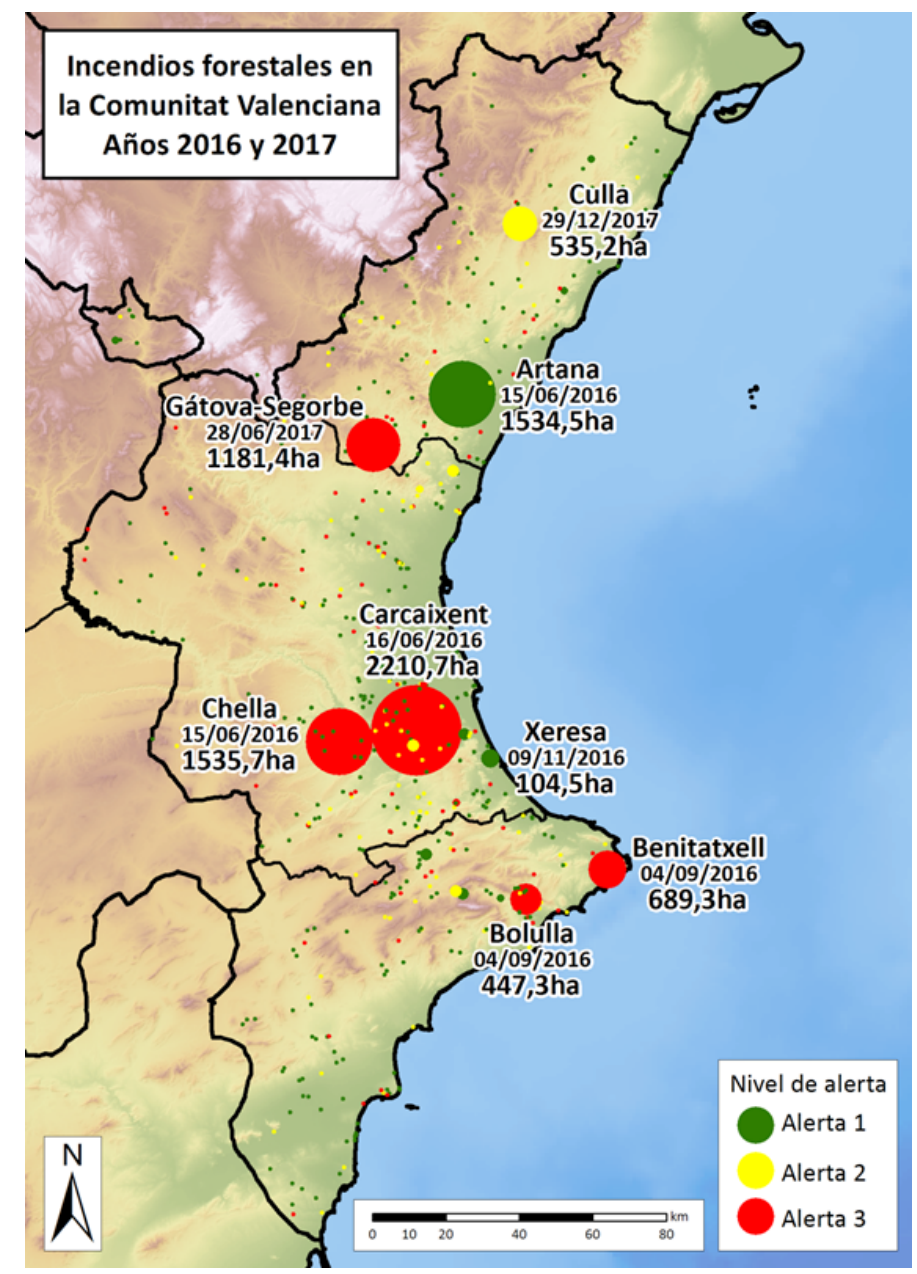




\subsection{Resultados mensuales del estudio estadístico}

Como muestran las figuras 6 y 7 , hubo un incremento del número de incendios los meses estivales, tanto en días con alerta 1 como en días con alertas 2 y 3 . También se incrementó notablemente la superficie quemada en verano. Los mínimos se produjeron en invierno. Aunque por el hecho de haber más días de alerta 1 se produjo un mayor número de incendios con dicha alerta, estos afectaron a una superficie mucho menor que los de alerta 3.

La figura 8 permite analizar los datos del número de incendios teniendo en cuenta las diferencias existentes en el número de días de alerta. Se aprecia como los días con alerta 3 , pese a ser pocos a lo largo del año, fueron los días en los que más incendios se produjeron. Esto indica que el índice PIF-GPV funciona correctamente y otorga los valores máximos de peligrosidad a los días en los que realmente se acaban produciendo más incendios debido a las condiciones meteorológicas propicias para ello. Si se analizan otras variables mensuales como la superficie quemada por incendio o la superficie quemada en función de los días de alerta, se obtiene la misma conclusión, pues con Alerta 3 las estadísticas fueron mucho peores.

Resultados de los meses de verano: por último, se han agrupado los meses comprendidos entre junio y septiembre para extraer la estadística general de los meses estivales. Se constata que, aunque predominaron los días de alerta 1 , se incrementaron los días de alertas 2 y 3 respecto al resto del año, especialmente en las zonas del interior debido a la mayor recurrencia de los vientos de poniente y de las bajas humedades relativas. En el litoral, en cambio, debido al predominio de las brisas, las alertas 2 y 3 fueron menos habituales. Las diferentes estadísticas sobre la gravedad de los incendios también muestran un incremento durante los meses de verano, aunque se observa una singularidad: debido al gran incendio de Artana (zona 1S, Castellón) en julio de 2016 y con pronóstico de alerta 1, las estadísticas estivales con alerta 1 fueron peores que con alerta 2. En el apartado 3.4. se analiza detalladamente este incendio.

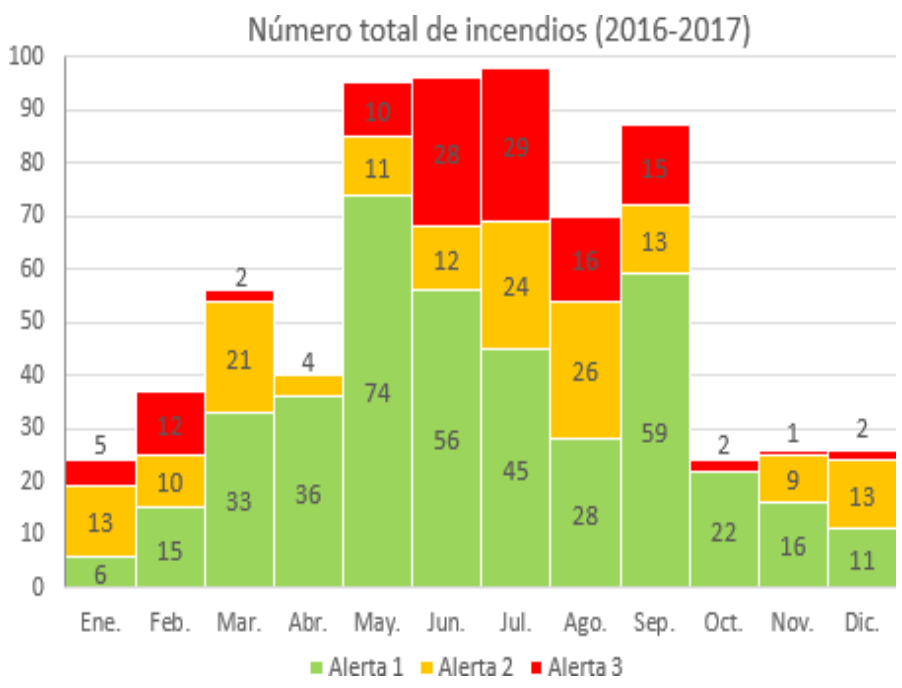

Figura 6. Número total de incendios forestales con las distintas alertas. Datos mensuales.

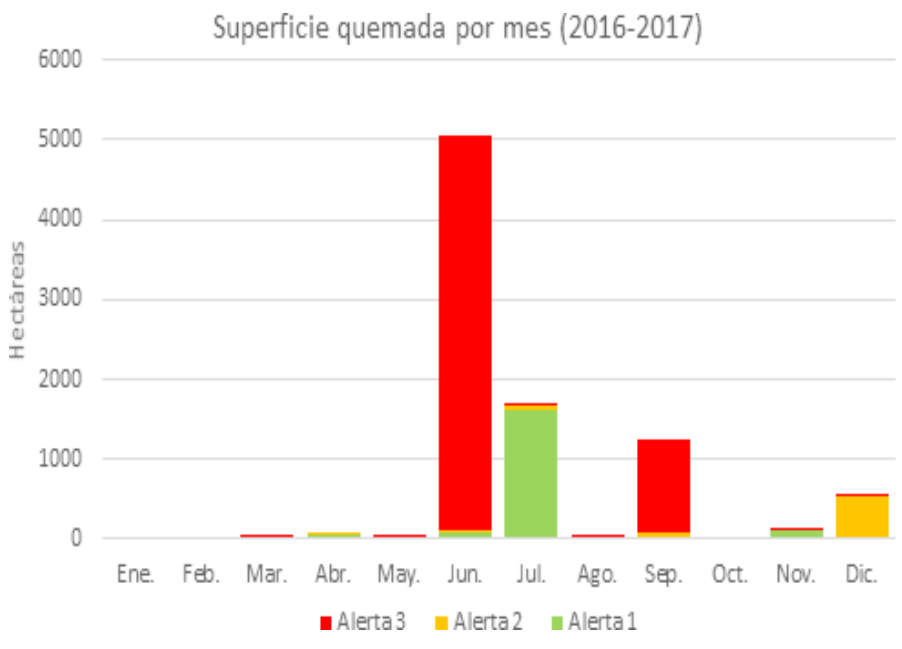

Figura 7. Superficie quemada (en hectáreas) con las distintas alertas. Datos mensuales.

Número de incendios por día de alerta (2016-2017)

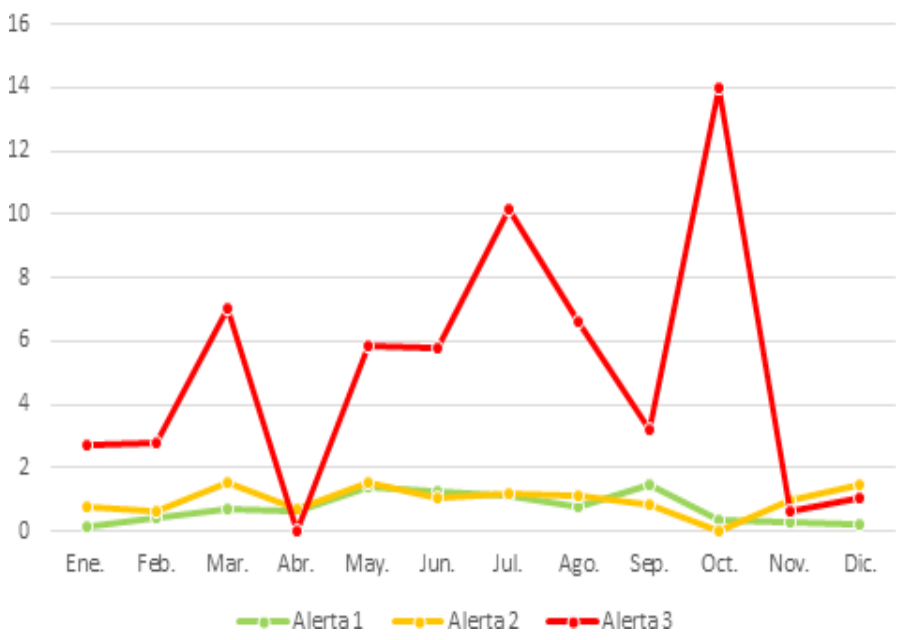

Figura 8. Número de incendios en función de los días de alerta. Datos mensuales. 


\subsection{Resultados del estudio estadístico según la metodología}

El índice experimentó dos cambios de metodología que influyeron en su funcionamiento:

- Cambio del día de predicción: hasta el 28/02/2017 se calculaba cada día el índice PIF-GPV para el mismo día de la predicción y para el día siguiente. Desde el 01/03/2017 se calcula para el día posterior a la predicción y para dos días después. Esta modificación se produjo con la finalidad de difundir las alertas con mayor antelación.

- Automatización del cálculo del índice: en junio de 2017 se automatizó la entrada de datos meteorológicos para el cálculo del índice incorporándolos directamente de las salidas del modelo de predicción.

Se han analizado y comparado los resultados globales obtenidos para periodos de tiempo similares de 2016 y 2017, antes y después de cada uno de estos dos cambios de metodología. La conclusión obtenida es que los resultados son similares, pues ninguno de los cambios muestra un empeoramiento de la calidad de la predicción. Así pues, se trata de modificaciones positivas porque la primera facilita la difusión de los niveles de alerta a la sociedad y los medios de prevención, y la segunda elimina cualquier posible fallo humano que pudiera darse.

\subsection{Incendio forestal de Artana}

El 25/07/2016 se inició un incendio forestal en el término municipal de Artana (zona 1S, Castellón). La superficie total afectada fue de 1534 hectáreas y el índice de peligrosidad pronosticado era 1 (medio-bajo). Este incendio ha influido en el estudio, ya que los resultados obtenidos con alerta 1 son peores a los esperados. ¿Qué sucedió para que se produjera un gran incendio con alerta 1? La situación meteorológica no fue de vientos terrales, y se desarrollaron las brisas durante los días del incendio. Algunos datos de las EMA (estaciones meteorológicas de AEMET) cercanas y correspondientes a la zona $1 \mathrm{~S}$ el 25/07/2016 fueron los siguientes:

- Atzeneta del Maestrat: HR mín.: $34 \%$ / Racha máx.: 40 km/h / Tx: $33^{\circ} \mathrm{C}$.

- Pantano de Arenós: HR mín.: $36 \%$ / Racha máx.: 40 km/h / Tx: $31^{\circ} \mathrm{C}$.

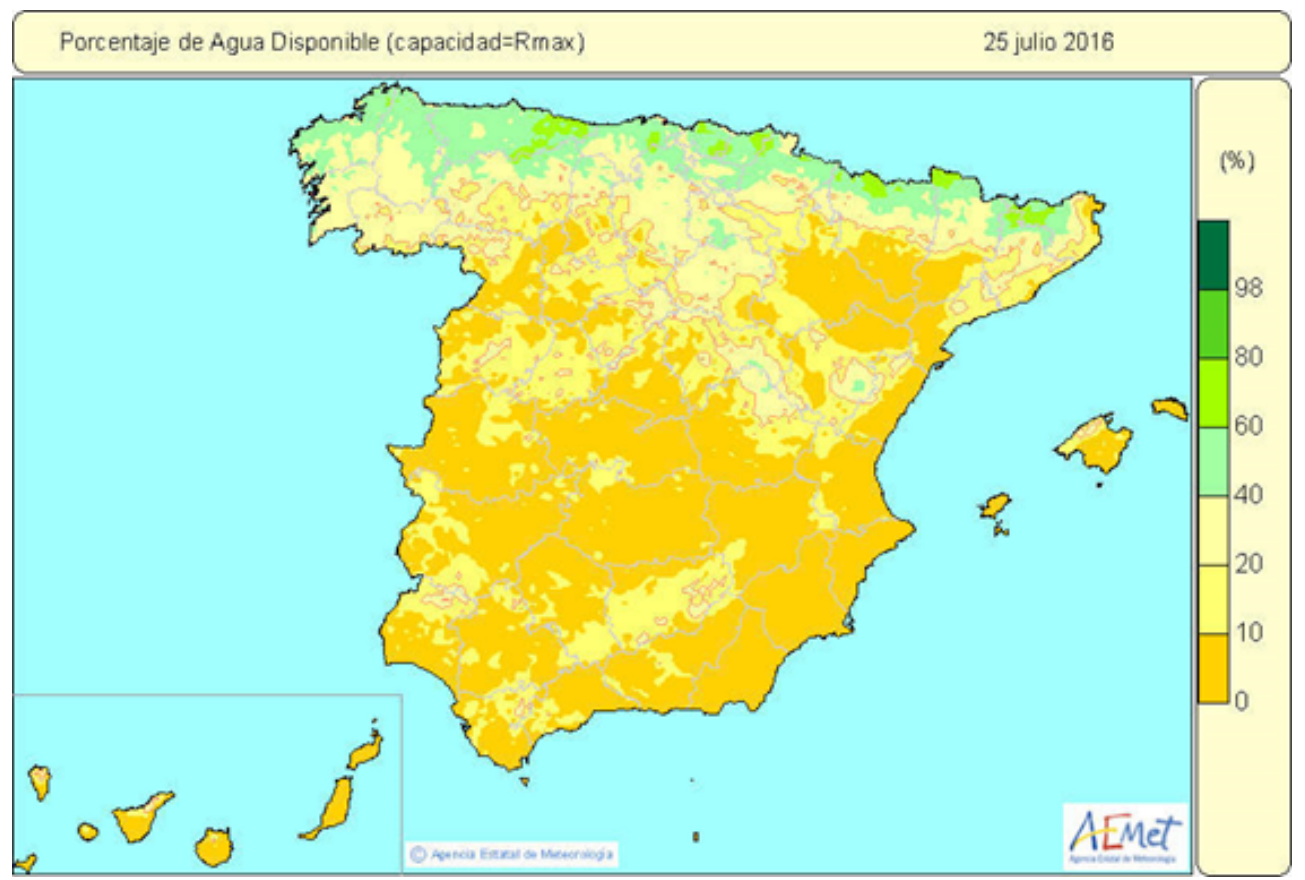

Figura 9. Mapa de porcentaje de agua disponible en el suelo en España. Fecha: 25/07/2016. 
Teniendo en cuenta los datos de observación el nivel de alerta sería 2, pero el estudio estadístico se ha realizado para los niveles de alerta comunicados a la Generalitat, y por tanto corresponde a una alerta 1 que es la que se pronosticó. Los valores observados son registros habituales en verano. Por ello, para explicar la magnitud del fuego hay que tener en cuenta otros factores.

Entre los factores meteorológicos destaca la escasa humedad del suelo en esa fecha. Como se puede observar en la figura 9 , la humedad del suelo en el sur de Castellón era inferior al $10 \%$. Las condiciones de humedad del suelo responden a anomalías de precipitación a escalas relativamente cortas. Los índices de precipitación, que evalúan la situación a más largo plazo en base a las precipitaciones acumuladas, se muestran en la figura 10.

En la figura 10 se aprecia como en julio de 2016 el índice estaba muy por debajo de la media para los periodos de 1, 2 y 3 años. Castellón sufrió una importante sequía a partir de 2013 y hasta

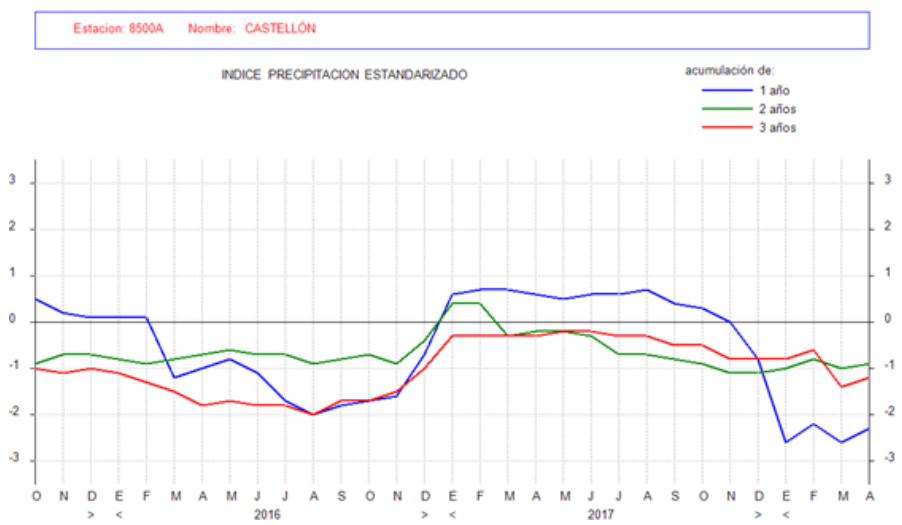

Figura 10. Índice de precipitación estandarizado para Castellón. Datos mensuales de acumulación a 1, 2 y 3 años del periodo comprendido entre octubre de 2015 y abril de 2018. diciembre de 2016, solo interrumpida por un mes muy lluvioso (marzo de 2015). Analizando los valores de este mismo índice para periodos de 1, 3 y 6 meses se constata que también estaban muy por debajo de los valores normales. Con lo cual, en el verano de 2016 la vegetación tenía un fuerte déficit de precipitaciones. La sequedad del combustible facilitó que el incendio fuera de grandes dimensiones pese a que la situación meteorológica era normal para un día de verano. La explicación de que fuera ese incendio el que adquiriera tales proporciones y no otro, en ese día y zona, hay que buscarla en factores no meteorológicos (la causalidad, la gestión del incendio, el tipo, cantidad y distribución de los combustibles, etc.).

\section{MODIFICACIÓN DEL ÍNDICE PIF-GPV}

\subsection{Metodología de cálculo del índice PIF-GPV}

La metodología utilizada hasta ahora, denominada PIF-GPV93 (MEDiAVILLA et al., 1993), consiste en el cálculo del índice PIF-GPV mediante una fórmula que otorga unos valores de peligrosidad basados en las predicciones automáticas del modelo meteorológico ECMWF para unas localidades de referencia de cada zona PREVIFOC, coincidentes con las EMAde AEMET. Para cada localidad se realiza a uno y dos días una predicción de los siguientes parámetros: temperatura máxima $\left({ }^{\circ} \mathrm{C}\right)$, humedad relativa mínima del aire $(\%)$, dirección del viento (decenas de grado) y racha máxima del viento $(\mathrm{m} / \mathrm{s})$. Esto implica que, a mayor temperatura, menor humedad relativa del aire, mayor racha máxima de viento y dirección terral del viento, se obtenga un valor de peligrosidad más elevado. Los diferentes rangos de estos valores previstos para su asignación a cada tipo de alerta son los siguientes: <40: peligro medio-bajo (alerta 1); 40-59: peligro alto (alerta 2); y $\geq 60$ : peligro extremo (alerta 3); siendo por tanto los umbrales 40 y 60 .

El último paso del proceso consiste en la asignación, por parte del predictor del GPV, de las alertas para cada una de las zonas. Además de los valores de peligrosidad otorgados por el índice, también puede tener en cuenta según su criterio: la probabilidad de precipitación y de tormenta (\%) según ECMWF; y los datos del balance hídrico de AEMET como la humedad del suelo y las precipitaciones registradas. Pero hasta el momento este último paso ha sido realizado de forma manual y no automática, de forma que existe una mayor probabilidad de que la precisión en la asignación del índice no sea tan elevada. 
a)

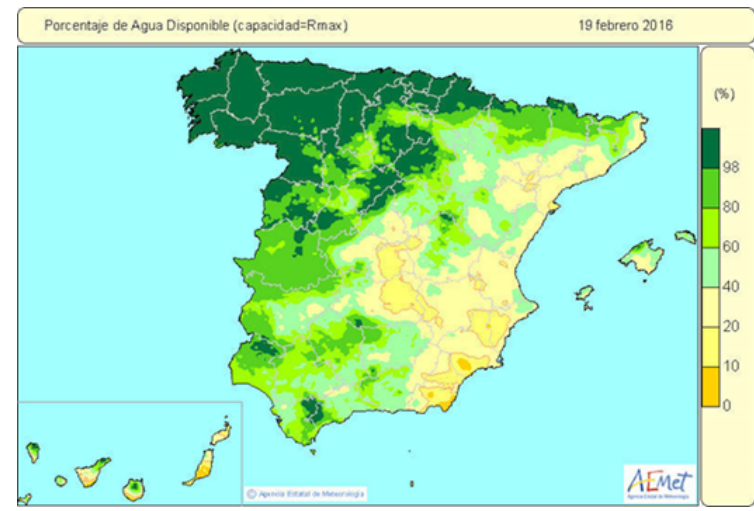

b)

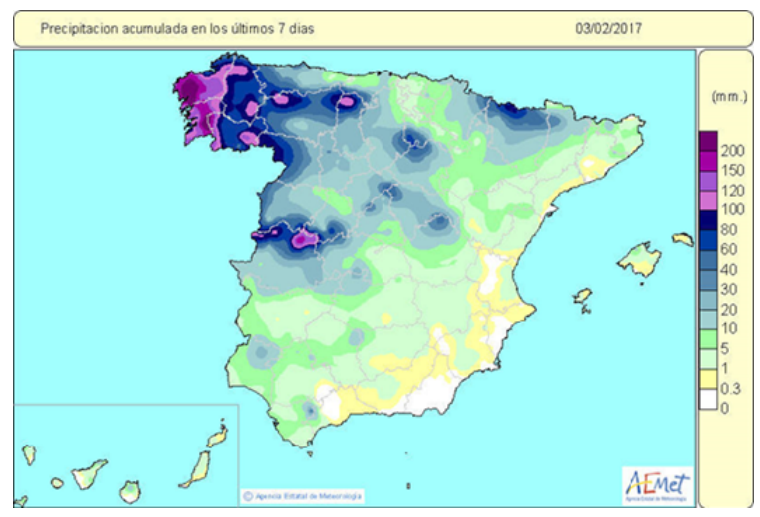

Figura 11. Ejemplos de mapas elaborados por el Servicio de Aplicaciones Agrícolas e Hidrológicas de AEMET: a) porcentaje de agua disponible según la capacidad del suelo -Rmáx-y

b) precipitación acumulada los 7 días anteriores.

El objetivo del trabajo ha consistido en elaborar una nueva metodología (PIF-GPV18) basándose en la metodología PIF-GPV93. Tras realizar el estudio del funcionamiento del índice PIF-GPV se ha considerado que la automatización en el cálculo del índice de parámetros como la humedad del suelo y la precipitación acumulada en los últimos 7 días podría optimizar los resultados obtenidos. El proceso llevado a cabo ha consistido en la extracción de los datos numéricos de humedad y precipitación de los mapas de balance hídrico, en su posterior correlación con los datos de incendios forestales y, por último, en la modificación de la fórmula del índice PIF-GPV.

\subsection{Resultados de la correlación}

Como se observa en la figura 12.a, el número de hectáreas quemadas por día fue muy elevado para los valores de humedad del suelo inferiores al $20 \%$, especialmente los días con alerta 3 . A partir del $20 \%$ se observa una disminución drástica de la superficie quemada que se hace aún más patente por encima del $50 \%$. A partir del $90 \%$ de humedad del suelo no se produjo ningún incendio en el periodo de estudio. Cabe destacar que el máximo que se observa del 16-20\% se debe a dos grandes incendios en la zona 3 en junio de 2016, pocas semanas después de un episodio de lluvias, de modo que el suelo aún conservaba algo de humedad.

En la figura 12.b se constata que la ausencia de precipitaciones en los días previos al incendio es fundamental para explicar la magnitud de los mismos. Así pues, según los datos analizados, los incendios más importantes se produjeron tras más de una semana sin llover, mientras que, con solo $5 \mathrm{~mm}$ de lluvia acumulados, los valores fueron cercanos a las 0 ha/día.

a)

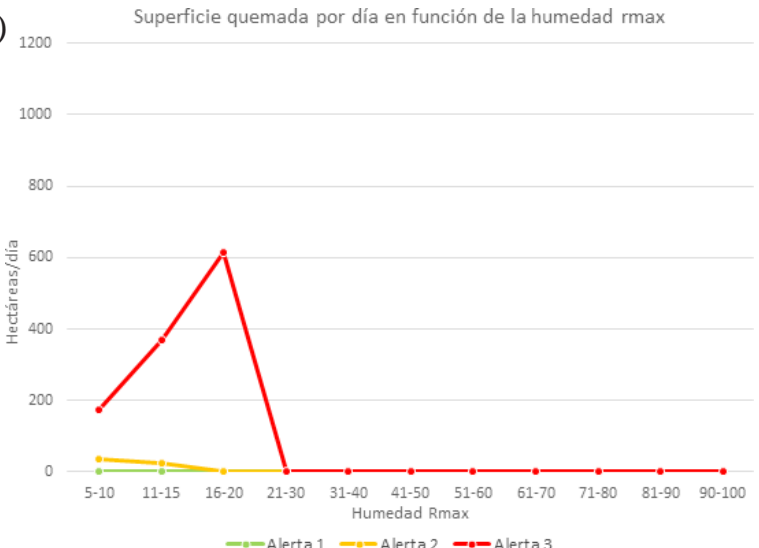

b)

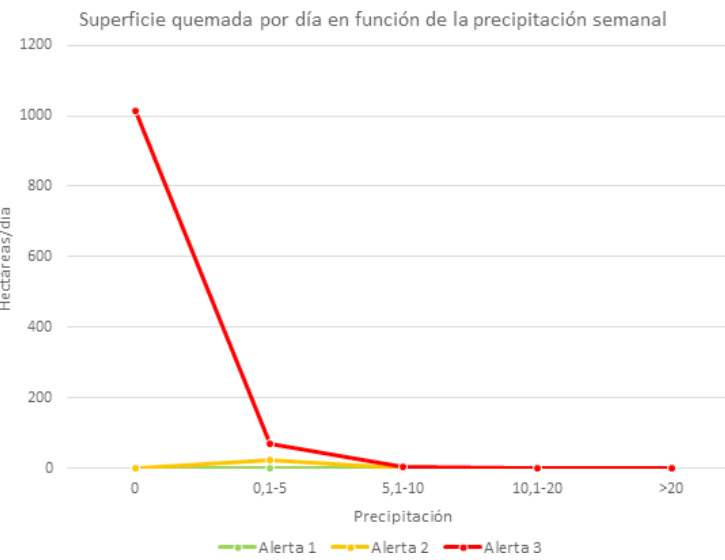

Figura 12. Superficie quemada por día (en hectáreas) en función de los valores diarios de: a) humedad del suelo (Rmáx); y b) precipitación semanal (acumulada en los 7 días previos). 


\subsection{Introducción de los parámetros en el cálculo del índice PIF-GPV}

Una vez estudiada la correlación se ha buscado la forma de introducir estos nuevos parámetros de forma lógica y argumentada, y tras realizar diversas pruebas con distintos ajustes, se ha elegido una relación exponencial, de la siguiente manera:

$$
\frac{\text { Sup }}{\text { Día }}=a \cdot e^{b \cdot I+c \cdot\left(\bar{H}_{S}-H_{S}\right)+d \cdot(P p)}
$$

que operando queda como:

$$
\operatorname{In}=I+\frac{c}{b} \cdot\left(\bar{H}_{S}-H_{S}\right)+\frac{d}{b} \cdot P p
$$

donde:

- Sup/Día es la superficie quemada por día en hectáreas;

- In es el índice de incendio PIF18;

- I es el índice de incendio PIF93;

- $\bar{H}_{S}$ es el porcentaje promedio de agua disponible en el suelo respecto al A.D.T. Rmáx;

- $H_{S}$ es el porcentaje de agua disponible en el suelo respecto al A.D.T. Rmáx;

- $P p$ es la precipitación acumulada en los 7 días anteriores;

- $a, b, c, d$ son constantes de ajuste.

Así pues, se encuentra una relación lineal entre el nuevo índice y el antiguo, incorporando los dos nuevos parámetros (humedad del suelo y precipitación). Se toma como $\bar{H}_{S}=20 \%$. Realizando el cálculo de regresión se obtiene:

$$
\frac{c}{b}=0,8 \frac{d}{b}=1,2
$$

Los diferentes rangos de los valores de peligrosidad previstos para su asignación a cada tipo de alerta se mantienen igual que hasta la fecha.

\subsection{Implementación del índice PIF-GPV18}

El nuevo método de cálculo del índice PIF-GPV se implementó a finales de julio de 2018 y desde entonces se encuentra operativo.

En la figura 13 se muestra la salida que genera la aplicación de cálculo del índice. Se incluyen las columnas nuevas de HS (humedad del suelo) e índice PIF-GPV18.

La figura 14 se corresponde a un ejemplo de cómo puede variar el índice antes y después de la modificación de su cálculo. En este caso se observa que se produjo un cambio del valor del índice en las zonas $1 \mathrm{~N}$ y 6 . En el primer caso, una humedad del suelo más alta hizo que los valores del PIF-GPV18 descendieran y fueran inferiores a 40, quedando con alerta 1 un día que con el PIF-GPV93 sería de alerta 2. Lo contrario sucedió en la zona 6: la escasa humedad del suelo y la ausencia de precipitación en Alicante elevaron el índice PIF-GPV18 por encima del umbral 40 (alerta 2), mientras que con el PIF-GPV93 el valor previsto era de 35 (alerta 1). 


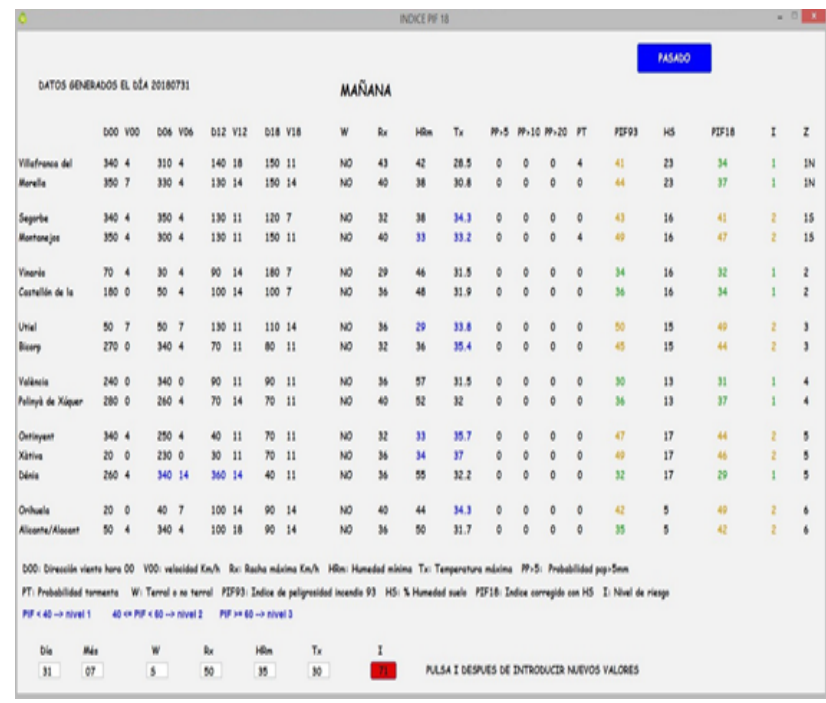

Figura 13. Aplicación que calcula el índice PIF-GPV tras su modificación.

\begin{tabular}{|c|c|c|c|c|c|}
\hline \multicolumn{6}{|c|}{ DATOS GENERADOS EL DIA 20180731} \\
\hline & PIF93 & HS & PIF18 & $\mathbf{I}$ & $z$ \\
\hline Villafranca del & 41 & 23 & 34 & 1 & $1 \mathrm{~N}$ \\
\hline Morella & 44 & 23 & 37 & 1 & $1 \mathrm{~N}$ \\
\hline Segorbe & 43 & 16 & 41 & 2 & is \\
\hline Montane jos & 49 & 16 & 47 & 2 & 15 \\
\hline Vinarò̀s & 34 & 16 & 32 & 1 & 2 \\
\hline Castellín de la & 36 & 16 & 34 & 1 & 2 \\
\hline Utiel & 50 & 15 & 49 & 2 & 3 \\
\hline Bicorp & 45 & 15 & 44 & 2 & 3 \\
\hline Volèmeia & 30 & 13 & 31 & 1 & 4 \\
\hline Polinyà de Xúquer & 36 & 13 & 37 & 1 & 4 \\
\hline Ontimyent & 47 & 17 & 44 & 2 & 5 \\
\hline Xàtiva & 49 & 17 & 46 & 2 & 5 \\
\hline Dénia & 32 & 17 & 29 & 1 & 5 \\
\hline Orihuela & 42 & 5 & 49 & 2 & 6 \\
\hline Alicante/Alacant & 35 & 5 & 42 & 2 & 6 \\
\hline
\end{tabular}

Figura 14. Ejemplo de variación del índice (31/07/2018).

Se ha realizado un análisis del funcionamiento del índice PIF-GPV18 para una situación concreta tras la implementación del mismo: el gran incendio de Llutxent (zona 5, Valencia) que se originó el día 6 de agosto de 2018 y se prolongó varios días, afectando a varios municipios y quemando más de 3000 hectáreas. Fue el incendio más grande del verano de 2018 en la Comunitat Valenciana. Los valores meteorológicos y los valores del índice PIF-GPV previstos (tanto PIF-GPV93 como PIF-GPV18) del día 5 de agosto para el día 6 se reflejan en la tabla 1.

\begin{tabular}{|lccccccc|}
\hline Localidad & $\begin{array}{c}\text { Viento } \\
\text { terral }\end{array}$ & $\begin{array}{c}\text { Racha } \\
\text { máxima }\end{array}$ & $\begin{array}{c}\mathrm{HR} \\
\text { mínima }\end{array}$ & Tx & PIF-GPV93 & HS & PIF-GPV18 \\
\hline ONTINYENT & NO & $36 \mathrm{~km} / \mathrm{h}$ & $23 \%$ & $36,7{ }^{\circ} \mathrm{C}$ & 56 & $5 \%$ & 63 \\
\hline XÀTIVA & NO & $36 \mathrm{~km} / \mathrm{h}$ & $23 \%$ & $37,4^{\circ} \mathrm{C}$ & 57 & $5 \%$ & 64 \\
\hline DÉNIA & NO & $36 \mathrm{~km} / \mathrm{h}$ & $41 \%$ & $34,7{ }^{\circ} \mathrm{C}$ & 43 & $5 \%$ & 50 \\
\hline
\end{tabular}

Tabla 1: Predicción del Índice PIF-GPV del 05/08/2018 para el día 06/08/2018. Zona 5.

Como se puede apreciar en la tabla 1, la previsión para el día que se inició el incendio era de temperaturas elevadas y humedades relativas bajas, pero no se predecían ni rachas de viento intensas ni tampoco viento terral. Es por ello que el índice PIF-GPV93 previsto era equivalente a la alerta 2. Debido a la ausencia de precipitaciones y la sequedad del suelo, el valor del PIF-GPV18 se elevó por encima del umbral 60 en dos de las tres localidades de la zona 5. Para el día siguiente (07/08/2018) las condiciones previstas eran algo peores, pero en dos de las localidades de referencia no se alcanzaba este umbral con el PIF-GPV93, mientras que el PIF-GPV18 supuso que las tres localidades lo superaran.

Por todo ello, teniendo en cuenta que usando el PIF-GPV18 el día 6 toda la región prelitoral e interior de la zona 5 superaba el umbral de peligro extremo y el día 7 se superaba en toda la región, se decidió que ambos días se iba a emitir una alerta 3 para la zona 5. Fue la única de las siete zonas PREVIFOC de la Comunitat Valenciana en la que se predijo dicha alerta, constatando así que la modificación del índice PIF-GPV con la introducción en su cálculo de la humedad del suelo y la precipitación semanal fue clave para ello. 


\section{CONCLUSIONES}

Partiendo de los resultados del análisis realizado se ha verificado que el índice PIF-GPV93 funcionaba correctamente y que las alertas por incendios que se han activado durante estos años en la Comunitat Valenciana han sido razonablemente adecuadas. Prueba de ello es que se observa como la mayoría de días del año tienen alerta 1 y raramente se producen grandes incendios, mientras los pocos días anuales con alerta 3 presentan peores estadísticas en cuanto a número y magnitud de incendios forestales debido a las condiciones meteorológicas. Los meses con los incendios más graves son los estivales, aunque en cualquier mes se puede producir un gran incendio si las condiciones son propicias para ello, como ocurrió en el incendio de Culla en diciembre de 2017 (SORIANo et al., 2018). En cuanto a la distribución espacial hay que señalar que los fuegos de mayor magnitud ocurren en espacios forestales montañosos del prelitoral y del interior. Pese a que este análisis estadístico ha sido esencial para introducir modificaciones en el índice PIF-GPV, hay que señalar que solo se han analizado dos años y que con una serie estadística más larga se podrían caracterizar aún mejor el comportamiento de los incendios forestales en territorio valenciano y el funcionamiento del índice.

Debido a la correlación existente entre la magnitud de los incendios y los datos del balance hídrico (humedad del suelo y precipitación registrada) se han introducido estos parámetros en el cálculo del índice PIF-GPV. Tras la implementación del índice PIF-GPV18 se ha observado una mayor precisión del pronóstico, incrementando los valores del índice en situaciones con el suelo muy seco y sin haberse producido precipitaciones, y haciéndolos descender en la situación contraria. Para obtener conclusiones definitivas sobre en qué medida ha aumentado la precisión del cálculo del índice PIF-GPV, en el futuro se debería realizar un análisis similar con una serie de datos extensa, previa y posterior a la modificación del índice.

\section{REFERENCIAS}

Alcover ,V., Mediavilla, J., Tamayo, J. y Correa, B., 1994. Evaluación y verificación de la campaña de apoyo meteorológico a PREVIFOC-94. Nota técnica n. ${ }^{\circ}$ 7. Instituto Nacional de Meteorología, GPV de Valencia.https://drive.google.com/open?id=1_0JHNAiSglmN7uZlv52zbWcy2dcIFFQr.

Botey, R. y Moreno, J. V., 2012. Metodología para estimar la humedad del suelo mediante un balance hídrico exponencial diario. Agencia Estatal de Meteorología. http://www0.aemet.es/wwl/balance2/metodo/ Metodologia.pdf.

Del Hoyo, J., 2014. Matriz de predicción por localidades para 7 días, Agencia Estatal de Meteorología. http://www0.aemet.es/wwj/stapwww/postproceso/matrix/docu/Docu_MPL_7dias.pdf.

Mediavilla, J., Alcover, V., Tamayo, J. y Correa, B., 1997. Forest fire fighting meteorological aid in the Valencian Community: PIF-GPVV Index. $3^{\text {a }}$ European Conference on Applications of Meteorology, 145-147. ISSN 0072-4122 ISBN 3-88148-331-4.

Mediavilla, J., Alcover, V., Tamayo, J. y Correa, B., 1993. Índice meteorológico de peligrosidad de incendios forestales. Nota técnica n. ${ }^{\circ}$ 2. Instituto Nacional de Meteorología, GPV de Valencia. https:// drive.google.com/open?id=1vxQc8nTIIM2cqNqQw3MMfNrvakerkfrE.

PREVIFOC, 1994. Plan Sectorial de Prevención Contra Incendios, Generalitat Valenciana. http:// www.agroambient.gva.es/es/web/prevencion-de-incendios/prevencion-incendios.

Radín, C., Revert, A., Mediavilla, J. y NúÑEZ, J. A., 2018. Análisis de incendios forestales en la Comunitat Valenciana y modificación del índice meteorológico de peligrosidad de incendios forestales PIF-GPV. GPV de Valencia. https://drive.google.com/file/d/1EVzDIkWE9P5qHniLpBPeIY7BcFp8nr18/view?usp=sharing. 
Soriano, J., Botella, M. y Cervera, A., 2018. Informe post incendio Culla 29/12/2017. Generalitat Valenciana. http://www.agroambient.gva.es/documents/162905929/164504386/024_2017_IPost_IF_Culla_ 29_12_2017/1a9d694f-998e-4498-93e1-b6ffd7013764. 